\title{
Bärmig, Sven
}

\section{Dialektik als Methode}

Grenzen.Gänge.Zwischen.Welten. Kontroversen - Entwicklungen - Perspektiven der Inklusionsforschung. Bad Heilbrunn : Verlag Julius Klinkhardt 2022, S. 59-66

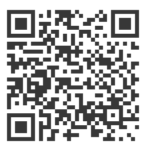

Quellenangabe/ Reference:

Bärmig, Sven: Dialektik als Methode - In: Grenzen.Gänge.Zwischen.Welten. Kontroversen -

Entwicklungen - Perspektiven der Inklusionsforschung. Bad Heilbrunn : Verlag Julius Klinkhardt 2022, S. 59-66 - URN: urn:nbn:de:0111-pedocs-238153 - DOI: 10.25656/01:23815

https://nbn-resolving.org/urn:nbn:de:0111-pedocs-238153

https://doi.org/10.25656/01:23815

in Kooperation mit / in cooperation with:

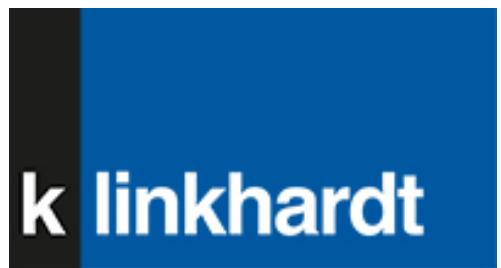

http://www.klinkhardt.de

\section{Nutzungsbedingungen}

Dieses Dokument steht unter folgender Creative Commons-Lizenz: http://creativecommons.org/licenses/by-nc-sa/4.0/deed.de - Sie dürfen das Werk bzw. den Inhalt unter folgenden Bedingungen vervielfältigen, verbreiten und öffentlich zugänglich machen sowie Abwandlungen und Bearbeitungen des Werkes bzw. Inhaltes anfertigen: Sie müssen den Namen des Autors/Rechteinhabers in der von ihm festgelegten Weise nennen. Dieses Werk bzw. der Inhalt darf nicht für kommerzielle Zwecke verwendet werden. Die neu entstandenen Werke bzw. Inhalte dürfen nur unter Verwendung von Die neu entstandenen Werke bzw. Inhalte dürfen nur unter Verwendung von
Lizenzbedingungen weitergegeben werden, die mit denen dieses Lizenzvertrages identisch oder vergleichbar sind.

Mit der Verwendung dieses Dokuments erkennen Sie die Nutzungsbedingungen an.

\section{Terms of use}

This document is published under following Creative Commons-License: http://creativecommons.org/licenses/by-nc-sa/4.0/deed.en - You may copy, distribute and transmit, adapt or exhibit the work in the public and alter, transform or change this work as long as you attribute the work in the manner specified by the author or licensor. You are not allowed to make commercial use of the work. If you alter, transform, or change this work in any way, you may distribute the resulting work only under this or a comparable license.

By using this particular document, you accept the above-stated conditions of use.

\section{Kontakt / Contact:}

\section{peDOCs}

DIPF | Leibniz-Institut für Bildungsforschung und Bildungsinformation Informationszentrum (IZ) Bildung

E-Mail: pedocs@dipf.de

Internet: www.pedocs.de

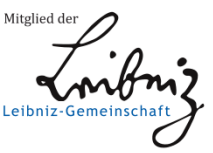




\section{Bernhard Schimek Gertraud Kremsner Michelle Proyer Rainer Grubich Florentine Paudel Regina Grubich-Müller (Hrsg.)}

\section{Grenzen.Gänge. Zwischen. Welten.}

\section{Kontroversen - Entwicklungen - Perspektiven der Inklusionsforschung}


Dieser Titel wurde in das Programm des Verlages mittels eines Peer-Review-Verfahrens aufgenommen. Für weitere Informationen siehe www.klinkhardt.de.

Bibliografische Information der Deutschen Nationalbibliothek Die Deutsche Nationalbibliothek verzeichnet diese Publikation in der Deutschen Nationalbibliografie; detaillierte bibliografische Daten sind im Internet abrufbar über http://dnb.d-nb.de.

2022.hg. () by Julius Klinkhardt.

Coverabbildung: Grafik: (C) Iris Kopera, Foto: kiyopayo/Adobe Stock.

Druck und Bindung: AZ Druck und Datentechnik, Kempten.

Printed in Germany 2022.

Gedruckt auf chlorfrei gebleichtem alterungsbeständigem Papier.

(c) (i)(3)(D) Die Publikation (mit Ausnahme aller Fotos, Grafiken und Abbildungen) ist veröffentlicht unter der Creative Commons-Lizenz: CC BY-NC-SA 4.0 International https://creativecommons.org/licenses/by-nc-sa/4.0/

ISBN 978-3-7815-5924-0 digital doi.org/10.35468/5924

ISBN 978-3-7815-2485-9 print 


\section{Inhalt}

Vorwort der Herausgeber*innen 9

Gertraud Kremsner, Bernhard Schimek und Michelle Proyer

Grenzen.Gänge.Zwischen.Welten.

Kontroversen - Entwicklungen - Perspektiven der Inklusionsforschung

\section{Kontroversen}

Georg Feuser

Die Bühne der Inklusion. Ein Prolog!

oder: Grenzgänge zwischen Welten

Mai-Anh Boger

Risse in der Landschaft der Inklusionsforschung -

Aktuelle Entwicklungen und offene Fragen

Sven Bärmig

Dialektik als Methode

Jan Jochmaring, Lena Bömelburg und Dirk Sponholz

Inklusive Berufsorientierung als Diskurs. Der ,scheinbare' Konsens:

gemeinsame Begriffe - unterschiedliche Ideen

Felix Kappeller

Prothetische Absenz, Articulating Gaps:

Repräsentationskritische Perspektiven auf visuelle Darstellungen

nicht an den Körper gefügter Prothesen

Jana York und Jan Jochmaring

Dilemmata einer inklusiven Arbeitswelt -

Menschen mit Behinderung zwischen Sondersystemen und

Gestaltungschancen einer Arbeitswelt 4.0

Juliane Gerland, Imke Niediek, Julia Hülsken und Marvin Sieger

Kontingenz von Differenzkonstruktionen in der inklusionsorientierten musikalischen Bildung am Beispiel des Umgangs

mit digitalen Musizier-Medien

Pierre-Carl Link

Zur Bedeutung einer befreiungspädagogischen Perspektive für die Inklusions- und Sonderpädagogik 
Katharina Hamisch und Robert Kruschel

Zwischen Individualisierungsversprechen und Vermessungsgefahr -

Die Rolle der Schlüsseltechnologie Künstliche Intelligenz in der inklusiven Schule

Felix Buchhaupt, Dieter Katzenbach, Deborah Lutz und Michael Urban

Zur Kontextualisierung der Inklusionsforschung

Lisa-Katharina Möhlen, Helena Deiß, Seyda Subasi Singh und Michelle Proyer

Lebenswelt(en der) Schule. Internationale Perspektiven zur Inklusion von

Schüler*innen mit Fluchterfahrungen. Eine Perspektive aus der Praxis

Thomas Hoffmann, Cathrin Reisenauer und Hendrik Richter

Helfen als individuelle Erfahrung und soziale Praxis zwischen

Deautonomisierung und Befähigung

\section{Entwicklungen}

Kathrin te Poel

Zur Bedeutsamkeit eigener schüler*innenbiografischer Erfahrungen von angehenden Lehrpersonen für den sich anbahnenden Lehrer*innenhabitus und seine Anerkennungsbezüge

Alina Quante und Oliver Danner

Grenzsetzungen bei Aufgabenbereichen von sonderpädagogischen und

allgemeinen Lehrkräften in inklusiven Settings

Edvina Bešić und Katharina Maitz

Das Boot: Eine Fluchtgeschichte -

Design-Based-Research in der Primarstufe

\section{Brigitte Kottmann}

Der Übergang von der Grundschule zur weiterführenden Schule als

(Soll-)Bruchstelle des Gemeinsamen Lernens

Anne Goldbach und Nico Leonhardt

Elemente von Macht im Kontext einer inklusionssensiblen

Hochschulentwicklung

Michaela Kaiser

Inklusionsbezogene Anforderungswahrnehmung -

Regulativ für (kunstpädagogische) Professionalisierung

Patrick Gollub, Silvia Greiten, Teresa Schkade und Marcel Veber

Schulpraktische Professionalisierung für den Umgang mit Heterogenität ein interdisziplinärer Blick aus hochschuldidaktischen Projekten 
Pierre-Carl Link, Cedric Steinert und Susanne Jurkowski

Implementierung von Inklusion als Querschnittsthema an der

Universität Erfurt durch das Kompetenz- und Entwicklungszentrum

für Inklusion. Inklusionsspezifische Professionalisierung der

Lehrer*innenbildung durch Team-Teaching, Fortbildung und

Online-Lernumgebung

\section{Perspektiven}

Timm Albers, Agnes Filipiak, Katja Franzen und Frank Hellmich

Kompetenzentwicklung im inklusiven Unterricht (KinU) -

eine internationale Perspektive

Katharina Maria Pongratz

Sehnsucht nach Bildung? Über den Einsatz systemischer Fragetechniken in der qualitativen Erwachsenenbildungs-/Weiterbildungsforschung bei Biografieträger*innen mit einer zugeschriebenen geistigen Behinderung

Laura Schwörer, Hannah van Ledden, Pia Algermissen und Mandy Hauser

Zusammenarbeit und Mediennutzung in einer

Partizipativen Forschungsgruppe

David Paulus, Patrick Gollub und Marcel Veber

Forschendes Lernen und Kasuistik. Grenzwelten und Zwischengänge

bezogen auf Reflexivität in der inklusionssensiblen Lehrer*innenbildung

Dietlind Gloystein und Ulrike Barth

Divers denken und handeln! - Theoretische Orientierungen und

Handlungsperspektiven für die Lehrkräftebildung

Ann-Christin Faix

Wie verändern sich die subjektiven Theorien von Lehramtsstudierenden über guten inklusiven Unterricht

Katja Baucke

Internationaler Vergleich als Reflexionsangebot. Eine explorative Studie zur Sicht von Hochschullehrenden auf schulische Inklusion in

Deutschland und Kanada

Bettina Amrhein, Benjamin Badstieber und René Schroeder

Zum Umgang mit als störend wahrgenommenen Handlungsweisen von

Schüler*innen in einem inklusionsorientierten Unterricht - Perspektiven

für die Lehrer*innenbildung (im Förderschwerpunkt emotionale und soziale Entwicklung) 
8 Inhaltsverzeichnis

Andrea Holzinger, Gerda Kernbichler, Silvia Kopp-Sixt, Mathias Krammer und Gonda Pickl

Profilierung für Inklusive Pädagogik (IP) im Lehramt der Primar- und

Sekundarstufe Allgemeinbildung

Timo Finkbeiner und Susanne Eibl

Kooperative Prozesse im technikbezogenen Unterricht 280

Autor*innenverzeichnis 287 


\section{Sven Bärmig}

\section{Dialektik als Methode}

\section{Methode}

Die folgenden Ausführungen sollen zeigen, dass mit der Dialektik eine ,Methode existiert, die sehr anregend und herausfordernd das Tagungsthema des ,Zwischen oder der ,Grenzen ' bearbeitet. Mit ,Methode` ist nach Jürgen Ritsert (2011) dabei eine einer bestimmten Ordnung folgende Weise des Denkens bei Theodor W. Adorno gemeint. Dessen dialektisches Verfahren analysiert Gesellschaft als Beziehung von Allgemeinem und Besonderem mit dem Primat der Gegenstände und Themen. Sie ist als Reflexion der Erkenntnisse angelegt und bezieht sich gleichfalls selbstkritisch auf die eigene Position. Insbesondere in komplexen und schwer durchschaubaren sozialen Verhältnissen wird dies bedeutsam (vgl. ebd.). Dann zeigt sich, dass bspw. eine Kategorie wie Inklusion nur scheinbar eindeutig ist und deshalb immer wieder dahingehend neu analysiert werden muss, ob sie das trifft, was damit ausgesagt werden soll. Allein aus dieser kurzen Hinführung ergeben sich einige wesentliche Merkmale dialektischen Denkens.

\section{Vom Begriff aus}

$\mathrm{Zu}$ bedenken ist, „dass die Begriffe immer schon etwas an uns heranbringen, was wir keineswegs erst stiften und dem wir uns in der Sprache selber gleichsam zu unterwerfen haben“ (Adorno 2015, 281). Dabei gilt zunächst: „Denken heißt Identifizieren“ (Adorno 2003, 17), was aussagt, mit einem Begriff auch eine bestimmte Sache zutreffend zu beschreiben/zu benennen.

Die Begriffe selbst lassen sich trotz ihrer Bestimmtheit sui generis nicht auf einen Inhalt ,ohne Weiteres' vereidigen, „das Bedürfnis der Definition“ (Adorno 2015, 281) verengt bereits. Dialektisches Denken ist deshalb für Adorno grundlegend verknüpft mit der Einsicht, dass die Gegenstände im Begriff nicht aufgehen und so in Widerspruch zur anvisierten Identifizierung geraten.

a. Verhältnis Begriff und Sache - Im begrifflichen Bemühen um den (historischen) Ursprung einer Sache, dem Ursprungsdenken, kommt es zur Verkennung, weil es eigentlich keine Phänomene gibt, die so statisch sind, dass sie als ewige Wahrheiten gelten könnten. Das hat immer einen Aspekt von der „Geschichtlichkeit der Welt (...), die grundsätzliche Erfahrung davon, dass es eigentlich nichts gibt, zwischen Himmel und Erde, was einfach so sei, sondern dass alles, was ist, 
eigentlich als ein Bewegtes und als ein Werdendes zu begreifen ist“ (ebd., 20). Gleiches gilt für das Telos: Auch ein endgültiges Ziel als ,Letztbegründung ist damit nicht zwangsläufig anzugeben bzw. ebenso problematisch. Die Kunst, vor die der Gebrauch der Begriffe die dialektische Methode stellt, ist also, das in jedem Begriff Enthaltene zu bewahren, es nicht abzuschneiden, nicht durch willkürliche Setzungen oder Festlegungen zu verdecken, aber es gleichzeitig so zum Bewusstsein zu erheben, dass es doch eben aus der Sphäre der Zweideutigkeit heraustritt. Diese nach Adorno innere Vermittlung zielt „auf den Sachverhalt, dass es Phänomene geben kann, die in einem strengen Gegensatzverhältnis zueinander stehen und bei denen dennoch der jeweils eine Pol den anderen (oder zumindest Merkmale des jeweils anderen) in sich enthält" (Ritsert 2011, 224). Eine Sache verweist zugleich auf das ihr Entgegengesetzte: Inklusion auf Exklusion, Bildung auf Dummheit ${ }^{1}$, Normalität auf A/Normalität, Zuschreibung von Behinderung auf Zuschreibung von Fähigkeiten; und zugleich ist Exklusion nicht gleich Inklusion usw. Dialektik ist damit „eine Veranstaltung des Denkens“ (Adorno 2015, 279), um „eben diesem Eigentümlichen des Begriffs gerecht zu werden, ihn weder als ein Unbestimmtes und Vages also zu nehmen, noch durch die Willkür der Definition ihn einfach abzuschneiden" (ebd.). In der ständigen Konfrontation des Begriffs mit der Sache, verstanden als immanente Kritik, gilt es, die begriffliche Definition als unzulänglich zu kennzeichnen. Bereits im Bemühen, genauer zu beschreiben, ergibt sich durchaus eine Änderung der Sache selbst (vgl. ebd., 18f.).

b. Verhältnis Allgemeines und Besonderes - Hinzu tritt das Verhältnis vom Ganzen als System und seinen Teilen, welches Adorno als komplex beschreibt und in dem das Einzelne nicht selbstständig (autonom) ist, weil: ohne das Ganze nicht zu denken, jedoch auch nicht durch die Totalität determiniert. Es ist nicht möglich, alles lediglich (begrifflich) in ein Ganzes (Theorie) einzufügen, „die Beschränktheit des Gedankens übersteigen und den Gedanken an seine richtige Stelle bringen“ (ebd., 53), sondern, „dass der Gedanke sich wirklich auf Gnade und Ungnade, unbarmherzig in die Dialektik selber hineinbegeben muss" (ebd.). Durch Denken des Teils ist das Ganze ebenso wenig bereits positiv gegeben wie umgekehrt, „sodass sie sich also unablässig fragen müssen, wie sie diese beiden Dinge eigentlich zusammenbringen“ (ebd., 129). Denn sie sind "nicht gleichsam zeitlos, dinghaft, gleichzeitig miteinander da“ (ebd.), sondern durch eine dynamische Beziehung charakterisiert. Für kritische Theorie heißt das bspw., dass Erkenntnis nie allein (Besonderes) stattfindet, sondern immer in einem größeren Zusammenhang (Allgemeines) gesehen werden

1 Horkheimer und Adorno (1989) sprechen von der ,Dummheit als Wundmal', etwas, was einer Person von anderen ,geschlagen ' wird. In diesem Sinne ist Dummheit als Ergebnis der Zueignung das Gegenteil von Bildung. 
muss. Auch Wissenschaft, und mit ihr Pädagogik und Inklusionsforschung, hat in einer spezifischen Gesellschaft eine spezifische Funktion. „Die Kontrolle wissenschaftlicher Urteile muss vielfach erfolgen, indem man Schritt für Schritt sich klar macht, wie man jeweils zum Urteil gelangte“" (Adorno 2003, 195).

c. Widerspruch - Widersprüche zeigen sich, da die Sache bzw. der Gegenstand nicht im Begriff, in der Definition aufgehen, sondern Beziehungen darstellen. Was der Begriff nicht fassen kann, das Nichtidentische, muss mitgedacht werden. „Kein Begriff, kein Urteil, kein System von gegenstandsbezogenen Sätzen kann die unendliche Mannigfaltigkeit der Merkmale jemals ausschöpfen, welche dem interessierenden Gegenstand zukommen und/oder zukommen könnten. Was ist, ist mehr, als es für uns ist und jemals sein kann" (Ritsert 2011, 228). Denn dort, wo die Dinge sich selbst gleichbleiben und miteinander identisch sind, besteht keine Notwendigkeit, die Gesetze der Entwicklung ${ }^{2}$ zu durchschauen, sondern nur dort, wo sie sich selbst widersprechen. Für die Dialektik gilt deshalb, die Unvermeidlichkeit der Widersprüche in der Sache selbst und das Weitertreibende der Widersprüche, die „Konsequenz des Gedankens" (Adorno 2015, 48), ernst zu nehmen. Widerlegung hat keine äußeren Gründe, die an den Begriff herangetragen werden, sondern das zu Erklärende (die Welt) ist in sich widersprüchlich, als Begriff für die widersprüchliche Praxis. Ein Gedanke, ein Prinzip, eine Definition, sich absolut setzend, ist bereits falsch. Definitionen, von Inklusion bspw., sind nicht durch Spielereien mit verschiedenen Ansichten - einen „sophistischen Meinungsstreit“ (ebd.) zwischen/unter Befürworter*innen oder Kritiker*innen - zu entkräften, sondern mit der Kritik der dahinterstehenden Erkenntnismethode. Wie wird Inklusion beschrieben, welche analytischen Kategorien werden benutzt? Welche Beziehungen zu anderen Begriffen werden hergestellt?33

2 Deshalb spricht materialistische Behindertenpädagogik (vgl. Jantzen 2007) davon, in Prozessen zu denken und nicht dinghaft. Das ist der Grund, weshalb die jeweilige Entwicklungssituation mit ihren Prozessen von Partizipation oder Isolation sowie die Zonen der aktuellen und nächsten Entwicklung im Mittelpunkt stehen. An der Entwicklungstatsache (vgl. Bernfeld 1973) gibt es kein Vorbei.

3 Als ,Paradebeispiel' sehe ich noch immer den Text ,Was ist Inklusion?‘ von Grosche (2015), in dem nicht einmal erwähnt wird, dass der Begriff aus der soziologischen Systemtheorie stammt und Exklusion als Komplementärbegriff zu verstehen ist. Stattdessen erfolgt die Darstellung einer historischen Abfolge von Begriffen (?), an deren Ende 28 Definitionen (!) vorgeschlagen werden, die verwendet werden können. 


\section{Konstellationen}

Im Aufeinander-verwiesen-Sein von Wahrnehmung (Forschung?) und Denken Letzteres ist erst die Reflexion des Denkens, die unbedingt notwendig ist - verortet Adorno die Idee von Subjektivität und Objektivität, die oftmals im falschen Sinne begriffen wird. Objektivität ist weniger wertneutrale denn möglichst umfassende Beschreibung und Analyse des Vorgefundenen ( $\mathrm{a}$ und b), wobei es die forschenden Subjekte sind, die ein Objekt beschreiben, analysieren, kritisieren. Zunächst heißt das, Erkenntnisoperationen und Sprachspiele machen Abstraktionen - das Abstrakte ist das für sich allein Stehende, ohne Beziehung - unvermeidbar. Daraus folgt, dass eine Beschreibung zum Referenten von Aussagen mit einer Fülle von Merkmalen und Beziehungen und daraus wiederum folgend von Schlüssen und Urteilen wird. Nur durch ein Subjekt (Forscher*in) ist ein Objekt erkennbar. „Wir beziehen uns ständig mit Sprache auf Themen (linguistisches Material), mit dem Geist auf geistige Phänomene. Doch daraus, dass uns alles Gegebene und Vorgegebene immer nur durch Empfindung, Wahrnehmung, Denken und Sprache gegeben sein kann, folgt logisch überhaupt nicht, dass alle Gegenstände Produkte unserer Empfindungen, unserer Gedanken, unserer Sprache wären“ (Ritsert 2011, 226). Hier laufen nach Ritsert verschiedene Themen und Thesen in Adornos Philosophie zusammen. Entscheidend ist, dass man sich nur dann einen klaren Begriff von Gegebenheiten machen kann, wenn man die Sensibilität für möglichst viel von dem entwickelt, woran der einzelne Begriff nicht heranreicht (Konsequenz aus Punkt a)). So wird das Nichtidentische zum Inbegriff dessen, was im ,jeweiligen System der Feststellungen nicht erfasst wurde und darin grundsätzlich nicht aufgehen kann" (ebd., 228).

Die einzelnen Wesenheiten sind als objektiver Zusammenhang (Totalität) zu erfassen, aber der objektiven Bestimmtheit nach als sich verändernd, als dynamische Prozesse, zu begreifen ${ }^{4}$. Vermittlung, das Verhältnis von Sein und Werden, gilt es ernst zu nehmen und damit ist die ,absolute Wahrheit " - bspw. ,Inklusion war schon immer dies oder jenes oder soll dies und jenes sein ' - ausgeschlossen. Wahrheit als objektives Kriterium entspricht keinem Einzelmoment der Erkenntnis und keine Einzelerkenntnis kann ihre ganze Wahrheit einlösen, sondern Erkenntnis und Wahrheit sind aufeinander verwiesen (vgl. Adorno 2015, 281). Wahrheit gibt es entsprechend „nur im Prozess, in der Konstellation der Momente, nicht in der Reduktion auf ein Urprinzip“ (ebd., 157) oder ein letztgültiges Telos. In dieser Konstellation richtet sich Dialektik als Kritik an der Erkenntnismethode

4 Diesen komplexen Zusammenhang hat Wolfgang Jantzen (2005) im Anschluss an die kulturhistorische Schule der sowjetischen Psychologie immer wieder beschrieben. Als kurze Einführung hier am besten: „Die Funktion des Ganzen ist aufgebaut als integrierende Tätigkeit, der hochdifferenzierte, hierarchisch verbundene, dynamische interzentrale Beziehungen zu Grunde liegen“ (ebd., o.S.). 
gegen eine geradlinige kontinuierlich verlaufende Entwicklung, sieht die Sachen selbst als widersprüchlich.

Inklusion (und Exklusion) als gesellschaftstheoretische Kategorie(n) können als angemessen betrachtet, ihr analytischer Gehalt muss immer wieder neu herausgestellt werden. Damit wird bedeutsam, ob ich im Zusammenhang mit Schule Begriffe wie ,Mündigkeit', ,Bildung', ,Lehren', ,Lernen` benutze oder ,Kompetenz', ,Sonderpädagogischer Förderbedarf ', ,Classroom-Management' oder aber, ob mit ,Teilhabe' dasselbe gemeint ist wie mit ,Inklusion'?

So wird Dialektik als Methode zu einer Sache der Erkenntnistheorie. Dieser Zusammenhang ist historisch und systematisch verschieden. Es kann Fortschritte und Rückschritte der menschlichen Erkenntnisfähigkeit in der Theorie, in den Begriffen geben. Die Zerlegung der Erkenntnis ist nicht schon die Erkenntnis selbst. Wir müssen Adorno zufolge die Beschränkung durchmachen, dass die kritische (Denk-)Bewegung bereits für die Wahrheit gehalten wird. Aber nur in der Bewegung, Vermittlung ist so etwas wie Wahrheit, Entstehendes, Entspringendes möglich. Es geht daher nicht (ausschließlich) um die Falschheit eines Satzes (einer Definition), sondern um dessen (deren) erkenntnistheoretischer Unzulänglichkeit und damit auch darum, die Erkenntnismethode selbstkritisch zu reflektieren: „Erkenntnisgewinn als Naivitätsverlust“ (Steinert 2007, 177). Das ist mit ,Bewegung' gemeint, denn ,Falschheit' ist wiederum nichts Zufälliges, wir geraten „in die Falschheit notwendig“" (Adorno 2015, 47) hinein.

\section{Materialismus oder Verhältnis Theorie - Praxis}

Die Konstellation der Begriffe und die Vermittlung von Allgemeinem und Besonderem verweisen in einem weiteren Schritt auf ein materialistisches Theorie-Praxis-Verhältnis. Denn hier gilt: Wenn alles gleich bleibt, still gestellt ist, gibt es keine Entwicklung, keine Bewegung. Dieser auf Marx bezogene Materialismus meint eine „handelnde Veränderung der Welt“ (ebd., 127), da konkrete Substanzen, soziale Formen' (vgl. Hirsch 1995), die Welt ausmachen. Zu dieser Materie (Institutionen, Organisationen) tritt der Mensch in ein Verhältnis, sinnlich tätig, in dem er einerseits dadurch geformt wird, aber auch Urheber*in dessen ist, und beruht ebenfalls auf geschichtlichen Entwicklungsschritten. „Das Bewusstsein kann nie etwas anderes sein als das bewusste Sein und das Sein des Menschen ist ihr wirklicher Lebensprozess ... Nicht das Bewusstsein bestimmt das Leben, sondern das Leben bestimmt das Sein“" (Marx \& Engels 1846, 26 zit. nach Aufenanger 1993, 104). Deshalb ist es wichtig, wie die Menschen arbeiten (Arbeitsteilung) oder lernen, sich miteinander in Beziehung zu setzen (funktional über bestimmte Rollenzuweisungen wie Konsument*in, Konkurrent*in, Kolleg*in, Bürger*in, 
Schüler*in, Behinderte*r, Ausländer*in ...), sich zu bilden, kurz: Es geht darum, wie sie ihre Gesellschaft gestalten. Kritische Theorie schärft den Blick dafür, sich nicht zu behaglich einzurichten.

Für kritische Theorie ist dabei charakteristisch, die Bedingungen der Möglichkeiten zu analysieren und mit der Realität ins Verhältnis zu setzen. Im Widerspruch geht es um die unabgegoltenen Ansprüche bzw. kann man das auch als verpasste Möglichkeiten der Entwicklung benennen, bspw. warum Menschen noch ausgeschlossen, diskriminiert werden, weshalb Inklusion gescheitert ist und welche Gründe dafürstehen, dies zu behaupten? Heinz Steinert (2007) verweist darauf, dass Adornos Theorie „kritisiert (...), indem sie auf die Versprechungen wie die Versagungen der bürgerlichen Gesellschaft hinweist und die Rückfälle auch der Intellektuellen aufzeigt. Sie macht uns keine Vorschriften, sie warnt uns" (ebd., 141). Kritik ist dabei „ein Instrument der Analysis. Als solches zielt sie in erster Linie auf sorgfältige Unterscheidungen von Merkmalen, klare Abgrenzungen von Begriffen sowie auf Schlüssigkeit der Urteile“ (Ritsert 2011, 230). Kritisches Denken im Sinne Adornos muss sich „nicht nur von jenen verdinglichten sprachlichen und kognitiven Rastern befreien, hinter denen der Wille zur Macht, zur Kontrolle und der stumme Zwang der Verhältnisse stehen, sondern sich so weit wie nur irgend möglich für die ,Vielheit der Verschiedenen' (Adorno), also diejenigen Bestimmungen der interessierenden Sachverhalte offen halten, welche durch das jeweils benutzte begriffliche Ordnungsmuster nicht erfasst wird“ (Ritsert 2014, 12). Dies legt zwei Dinge nahe. Erstens widerspricht das einem Monismus, der alles erfassen kann, und zweitens dem Relativismus, der alles als gleichgültig betrachtet. Dialektik hat jedoch eine normative Dimension, immer dann, wenn durch das Nichtidentische die Zwangsmechanismen, Macht- und Herrschaftsverhältnisse offengelegt werden, der negative Zustand der Welt „bei seinem eigenen Namen" (Adorno 2015, 106) benannt wird. Denn die Frage der Humanität, also die Diskussion um die Bedingungen des Zusammenlebens, ihrer institutionellen Ausgestaltung und deren Auswirkungen auf das einzelne Individuum, bleibt immerwährende Aufgabe der Menschen. Dies gilt auch für die Inklusionsforschung und damit ebenso für die Fragen nach Mündigkeit und Bildung als Menschenrecht (vgl. Gruschka 2015).

Festzuhalten ist: Der negative Zustand der Welt lässt sich am individuellen Leid des einzelnen Individuums (Besonderes) festmachen und den bestehenden gesellschaftlichen Möglichkeiten (Allgemeines) gegenüberstellen. Kritische Theorie, und mit ihr dialektisches Denken als Methode, ist eine Instanz, die dazu verhilft, dies auszusprechen (vgl. Bärmig 2017). Dialektik, wie sie als ,Methode‘ hier vorgestellt wird, heißt also, die Begriffe zwar ernst zu nehmen, sie jedoch nicht als abzuarbeitende oder zu erreichende Definitionen stillzustellen. Es geht bspw. darum, ob ,Inklusion' tatsächlich das beschreibt, was damit gemeint ist, weil es im Besonderen für eine Person als Begriff selbst nicht hinreicht, oder darum, welche 
Teile der Gesellschaft Inklusion durch den Einschluss in eine Institution, also als Ausschluss, erfahren ${ }^{5}$.

Dabei ist - negativ formuliert - festzuhalten, was unterbleiben soll oder ,zu unterlassen ist', z.B. ,Einschluss ist nicht Inklusion'. Das Positive wiederum kann zwar anvisiert, doch nie festgehalten werden. Es würde zu einem statischen Phänomen, selbst wenn Veränderung stets eine „wie immer auch erklärungsbedürftige Vorstellung vom Richtigen" (Ritsert 2011, 232) braucht. Reflexivität ist ein zentrales Element dialektischen Denkens. Sie kommt nicht von außerhalb, sie erkennt den Herrschaftszusammenhang und ist vor allem der Anwendung nach in einem naiven Theorie-Praxis-Verhältnis entgegengesetzt, da Widerspruch und Nicht-Identität nicht im bloßen Denken aufhebbar sind (vgl. ebd., 228). Adornos Methodenverständnis, so Ritsert weiter, sein Denken und Schreiben, lässt sich mit „keiner metalogischen Position vereinbaren, die Form und Inhalt trennt, wenn nicht dichotomisiert. Er hält diese strikte Trennung - (...) - für schlechthin undurchführbar" (ebd., 223). Ritsert betont, dass es keine Schnittmenge mit einer ,zufällig' immer gleichen Aufteilung gibt, es nicht um einen Moderantismus als einheitlichen Standpunkt einer, harmonischen' Mitte, mengenlogisch betrachtet, geht. Was wäre auch die Mitte von Inklusion, Normalität oder Bildung?

\section{Literatur}

Adorno, Th. W. (2003): Negative Dialektik/Jargon der Eigentlichkeit. Frankfurt am Main.

Adorno, Th. W. (2015): Einführung in die Dialektik. Frankfurt am Main.

Aufenanger, J. (1993): Bertelsmann Lexikon Philosophie. Gütersloh.

Bärmig, S. (2017): Behindertenpädagogik und kritische Theorie. In: Lütje-Klose, B., Boger, M., Hopmann, B. \& Neumann, P. (Hrsg.): Leistung inklusive? Inklusion in der Leistungsgesellschaft. Band 1. Bad Heilbrunn, 127-138.

Bernfeld, S. (1973): Sisiphos oder die Grenzen der Erziehung. Frankfurt am Main.

Grosche, M. (2015): Was ist Inklusion? - Ein Diskussions- und Positionsartikel zur Definition von Inklusion aus Sicht der empirischen Bildungsforschung. In: Kuhl, P., Stanat, P., Lütje-Klose, B., Gresch, C., Pant, H. A. \& Prenzel, M. (Hrsg.): Inklusion von Schülerinnen und Schülern mit sonderpädagogischem Förderbedarf in Schulleistungserhebungen. Bielefeld, 17-39.

Gruschka, A. (2015): Verstehen lehren. In: Schnell, I. (Hrsg.): Herausforderung Inklusion - Theoriebildung und Praxis. Bad Heilbrunn, 223-232.

Hirsch, J. (1995): Der nationale Wettbewerbsstaat - Staat, Demokratie und Politik im globalen Kapitalismus. Berlin.

Horkheimer, M. \& Adorno, Th. W. (1989): Dialektik der Aufklärung - Philosophische Fragmente. Leipzig.

Jantzen, W. (2005): Was ist der Mensch? Konturen einer marxistischen Anthropologie. Online unter: http://www.basaglia.de/Artikel/Mensch-MBl-2005.pdf (letzter Zugriff: 23.02.2021).

5 Es ist erstaunlich, dass alle großen Träger der Behindertenhilfe in ihren schriftlich fixierten Leitbildern von Inklusion sprechen, Heime jedoch oftmals eher mit Exklusion in Verbindung zu bringen sind. Auch diese thematischen Widersprüche in verschiedenen Bereichen der Gesellschaft, die weniger frei sind als andere, sind charakteristisch für gesellschaftliche Entwicklung. 


\section{6 | Sven Bärmig}

Jantzen, W. (2007): Kritisch-materialistische Behindertenpädagogik. In: Greving, H. (Hrsg.): Kompendium Heilpädagogik. Band 2. Troisdorf, 86-95.

Ritsert, J. (2011): Methode. In: Klein, R., Kreuzer, J. \& Müller-Doohm, S. (Hrsg.): Adorno Handbuch. Leben - Werk - Wirkung. Darmstadt, 223-232.

Ritsert, J. (2014): Themen und Thesen kritischer Gesellschaftstheorie. Weinheim/Basel.

Steinert, J. (2007): Dialektik der Aufklärung als Forschungsprogramm. Münster. 